\title{
Weight Bias: Twitter as a Tool for Opening Dialogue among Broad Audiences
}

\author{
Emily P. Williams*, Shelly Russell-Mayhew, Sarah Nutter, Nancy Arthur, Anusha Kassan \\ Werklund School of Education, University of Calgary, Calgary, Canada \\ Email: *emily.williams@ucalgary.ca
}

How to cite this paper: Williams, E.P., Russell-Mayhew, S., Nutter, S., Arthur, N. and Kassan, A. (2018) Weight Bias: Twitter as a Tool for Opening Dialogue among Broad Audiences. Social Networking, 7, 111-125.

https://doi.org/10.4236/sn.2018.73009

Received: April 4, 2018

Accepted: June 30, 2018

Published: July 3, 2018

Copyright $\odot 2018$ by authors and Scientific Research Publishing Inc. This work is licensed under the Creative Commons Attribution International License (CC BY 4.0).

http://creativecommons.org/licenses/by/4.0/

\begin{abstract}
Twitter is a tool for strengthening research knowledge mobilization to the general public. In this article, we highlight how Twitter can be used to open social dialogue about research related topics between users from multiple perspectives, using the topic of weight bias; a cultural issue largely perpetuated by the media. Specifically, Twitter (@UCalgary Body BS) was used by an interdisciplinary research team to under line cases of global news, stories, and policy related to weight bias and/or weight-related issues for a broad audience to consume. We position Twitter as a relevant means for 1) shaping the research lifecycle, 2) increasing community participation and engagement regarding specific research topics, 3) co-creating evolving social dialogues and critique, 4) reaching broader audiences, 5) opening up sites of debate and tension within a topic, and 6) engaging with a topic salient within our society, a topic that saturates the media - weight bias.
\end{abstract}

\section{Keywords}

Twitter, Social Media, Social Networking, Weight Bias, Knowledge Mobilization, Opening Dialogue

\section{Introduction}

One of the most important aspects of the research life cycle is knowledge transfer. Historically, researchers have been expected to share the results of completed studies in outlets that demonstrate their academic scholarship, such as peer-reviewed journal articles and conference presentations. In other words, the results of research were primarily aimed for use by other academic researchers-essentially for the culture of academia ([1]). However, the diversity of stakeholders in research has changed and demands for increased community participation and access to research for public use have become commonplace. Knowledge in- 
tended for a private and/or monetized culture has been challenged, in favor of opening and sharing knowledge within a variety of cultural domains ([2]). Funders, including federal and provincial governments, are requiring that researchers improve the accessibility of their knowledge, including consumption by community agencies and the public ([3]). This concern has been raised in response to critique that publicly funded research needs to be accessible to the public. As a result, the benchmarks used for knowledge mobilization plans have shifted beyond the traditional academic outlets, to broader sources of distribution and ways to engage and promote open dialogue between researchers and the communities in which they live and work, through formal and informal communication ([1] [2]). Such a shift holds across multiple disciplines and topics of study ([4]).

Social media forums, such as social-networking, and instant messaging, are now considered part of daily communication channels. The use of social media has the potential to increase the scope of possible audiences for knowledge mobilization about research and to increase innovative applications of such knowledge ([5]). Additionally, social media can be used to break down traditional outlets for networking and dissemination that are often organized as silos within specific disciplines, through distribution across academic and social boundaries. In other words, social media can be used as a tool to bridge the gaps between researchers in academia and communication with multiple stakeholders in the community ([6]) to inspire creative and innovative use of research knowledge ([5]). In this sense, the community potentially expands to include worldwide distribution, with unprecedented speed of delivering real-time data or results ([7] [8]). The limits of distribution are only as limited as consumer access to technology. As such, social media can be used to broaden the audience of interest in research across academic boundaries and foster interdisciplinary inquiry within the academy.

Beyond academic settings, social media can be used to enlist interest and participation in the research topic through open dialogue. A key point to note is that communication is not just a one-way process of researchers delivering information to the public. Rather, the use of social media can be employed to increase the input and accessibility of public voices available to academic researchers ([4]). Consequently, the use of Twitter is positioned as a knowledge mobilization and social networking device that can assist researchers to disseminate knowledge about research in a format and language that is often more user-friendly to the public ([9] [10]). Moreover, in addition to being a userfriendly modality, Twitter also allows researchers to receive feedback from their audiences and engage in candid conversations. Open dialogues via Twitter may demonstrate the tensions in the field regarding language or stakeholder perspectives, thus enriching researchers' understandings of the phenomenon they are studying.

The purpose of the paper is threefold. First, we discuss the use of social media 
to inform stages of the research life cycle, highlighting the use of Twitter. A key premise of the discussion is that knowledge mobilization through social media might be better viewed as a continuous forum for community engagement, as opposed to singular events that occur at the stage of completing a research study. Second, we offer how our interdisciplinary team chose and utilized Twitter to communicate the concept that weight is a social justice issue. We describe our Twitter account and its purpose as well as provide a collection of tweets and observed responses that occurred when we ran a continuous platform to create discussion around a research initiative on the topic of weight bias. Third, we seek to illustrate the power of social media, the nature of the Twitter responses, and the observation of diverse feedback that we received from the public. In this way, our Twitter account was used to create discussion of weight bias, a cultural issue, within the very medium that is largely used to perpetuate such negative attitudes-the media. We hope that our foray into the use of social media, including expected and unexpected responses from followers, as well as how the Tweets created space for reflection, deconstruction, critical thinking, and activism, will be of interest to other researchers who are new to using social media and social networking in research. Further, we aim to highlight how social media is transformative, as it moves knowledge beyond the academy and into the community.

\section{Social Media and the "Research Lifecycle"}

The use of social media in general has noteworthy implications at all points of the research lifecycle ([4]). Beginning with recognizing research opportunities, using social media assists researchers with pin-pointing "hot button issues" and other areas that are highly debated within specialized fields. Further, social media assists in the working lifecycle of research by gathering support and finding collaborators ([4]), either within internal or external communities. Next, social media tools fit into the researchers' work flow while reviewing the literature, collecting data, and analyzing the data. Researchers have access to all publicly available information, making it possible for researchers to collect and analyze a wealth of online information. Finally, social media has an application in knowledge dissemination, as it serves as a novel and informal way of sharing research findings, making them accessible to those who do not traditionally have access to academic, peer reviewed resources ([10]).

Regardless of the stage at which research information is shared through Twitter, it holds the potential to increase interdisciplinary networking and, in some cases, lead to collaborations that would not have otherwise developed ([4]). While researchers tend to present or publish the results of their scholarly efforts in specific spaces and places, a wider audience can be reached by using Twitter.

That is, researchers are able to move beyond their typical means of knowledge mobilization and engage in cyclic conversations with stakeholders across different disciplines and areas of interest. As such, Twitter allows for dialogue that 
would not be seen within a specific setting ([1]). This interdisciplinary communication can be helpful to researchers using Twitter, as various points of views are shared, taken for granted interpretations of topics are debated, and different directions as well as others' feedback may be integrated into future work. Moreover, it can be of interest to those conducting similar research in a different discipline, expanding conversations in differing levels and directions.

\section{How Social Media Is Used to Shape Research}

When social media is used to informally disseminate information, it functions as a constant feedback loop, providing researchers with invaluable real-time data. Specifically, Twitter provides research teams with instantaneous feedback, by way of users responding to, retweeting, hashtaging, and liking content online. Controversial content often attracts the most attention, thus serving as an indicator to researchers that particular topics are more salient than others ([4]).

Using Twitter in research. Twitter is an international service, allowing researchers to connect with users worldwide and at an exponential speed ([7] [8] [11]). Twitter has been described as the dominant microblogging service and is used by researchers, practitioners, and organizations ([4]), pointing to this platform's reputable communication and knowledge mobilization capabilities. Although the most popular social networking site is Facebook, professionals have noted that Twitter is more appropriate for work-related practices ([12]). Thus, Twitter serves as an efficient method for bridging research and academia to the community. The use of Twitter for research purposes allows for instantaneous debates and the sharing of ideas among users and between those who have limited or no access to academic resources ([10]). Biswas and Kirchherr ([6]) suggested that knowledge shared via Twitter allows for information produced within academic settings to be made accessible to others, with the added benefit of representing a "trusted voice". Further, social media can elicit feedback from people from a variety of backgrounds in community settings who might not otherwise have access to research ([10]).

Twitter has three distinctive features which aid in effortless communication: retweets, the use of @ followed by a username, and \# (hashtag). Twitter users can forward or share an existing Tweet by retweeting it, usually marked by a RT. Retweeting is a method of showing support for Twitter content, or sharing existing content and adding one's perspective to it. Second, by using @ followed by a username, Twitter users can be mentioned in Tweets and are alerted that they have been mentioned. Last, hashtags can be created by the use of \# followed by any term. Further, hashtags are commonly used at conferences and events, as they provide convenient ways of collecting information on the same topic and can be accessed in real time ([1]).

@UCalgaryBodyBS. Our research team, comprised of an interdisciplinary group of academics, post-doctoral fellows, and graduate students, launched a Twitter account as part of a research initiative on the topic of weight bias as a 
social justice issue. The research team assembled into a collaborative working group due to everyone's shared interests in weight related issues, education, health sciences, and the promotion of social justice. @UCalgaryBodyBS was created with the intent to (a) highlight key media campaigns, news articles, and events happening worldwide concerning weight related issues, (b) collect current and useful resources for teaching and learning about weight bias, (c) draw attention to the larger systemic issues at play within the media, and (d) connect the internal and external community around the issue of weight and social justice. The media watch was carried out by one graduate student research assistant for a duration of 1.5 years.

Why Twitter? Twitter was chosen as an ideal medium to share our research and objectives. Aside from disseminating information formally in academic, peer reviewed channels, our decision to share information informally through social media reflects a noteworthy shift towards more casual ways of knowledge distribution ([9] [10]). Further, Twitter was chosen as a social networking medium to advance the conversation on weight bias and related issues, as researchers from various specializations have approached weight bias from independent viewpoints and from conflicting theoretical orientations ([13]). Although social media continues to be flooded with negative attitudes and derogatory remarks about individuals with large bodies across social media platforms, posts that counter these negative attitudes and promote fat acceptance are also present ([14]), and were of particular interest to the researchers. It was our intention to use @UCalgaryBodyBS as a method of bridging diverse perspectives and connecting individuals from a wide array of backgrounds in making weight bias a salient topic of conversation. Therefore, our Twitter account served as a way to increase awareness of weight bias and associated discrimination, to promote size acceptance and body diversity, and to encourage dialogue across differing perspectives and professional backgrounds.

We proceeded to make sense of the data captured through Twitter by way of a case study-through observations, reflections, and discussions between the research team, and gained valuable insights into our topic of interest: weight bias. Weight bias is defined as the negative attitudes, stereotypes, and discrimination towards individuals with obesity ([15]). Weight bias was identified as a topic of interest by the research team given that it remains an overt, widely held, and expressible form of prejudice ([16] [17]). In addition, controversy exists within weight bias research as to the most appropriate language to use when referring to individuals with large bodies, as well as our understanding of body weight and explanations for weight bias ([13]). When considering weight bias as a social justice issue, the need for interdisciplinary effort is critical ([18]), as equity on a large scale cannot occur without progress across disciplines. Using Twitter allowed for multiple people to comment or offer their opinions about weight bias, making the conversation that may have once been stagnant or one-sided to become alive with complexity and nuance. It is partly from this diversity that social 
media functioned as a feedback loop to the research team regarding current attitudes about weight bias.

\section{Weight as a Social Justice Issue: Unveiling Weightism in Twitter}

Diverse perspectives regarding weight bias and weight-related topics have been noted in research, face to face conversations, and through social media platforms, alluding to the diversity of theoretical orientations and viewpoints towards this topic, and more generally towards individuals living in large bodies ([13] [14]). Weight bias has been declared the fourth most frequently reported form of discrimination, with an increase of $66 \%$ in its occurrence from 1995 to 2006 ([19]). Weight bias impacts individuals' quality of health care (e.g., [20]), success in education (e.g., [21]), success in the workplace (e.g., [22]), and interpersonal relationships (e.g., [23]). Sociocultural beliefs and stereotypes about individuals with large bodies are largely perpetuated through representation of individuals with large bodies in entertainment media ([24]), discussion on social media ([25]), and the framing of weight and obesity in news and public health campaigns ([26] [27]).

Multiple disciplines including health care, psychology, and fat studies recognize weight bias as a social issue, however these disciplines largely remain segregated, despite sharing a common goal ([13]). It was our intention to incorporate modern technology into our research and dissemination, and to utilize media to network with individuals who hold different perspectives. Twitter features such as retweets and hashtags provided the team with efficient ways of sharing information, allowing the team to informally interact and learn from others around the world, while collaboratively engaging in invaluable dialogue. We intended to demonstrate our evolved understanding of how weight bias is taken up by a broad array of stakeholders via social media, through the narratives of Twitter users. Specifically, observations of differences among diverse professionals and tensions within Twitter dialogues are emphasized and illustrated through Twitter excerpts.

The authors used Twitter as a means to bring attention to the prevalence of weight bias as a cultural issue, and to challenge normative discourses about the body on social media. In response to our tweets, users expressed explicit tensions, debates, and strong reactions, which served to demonstrate that the topic of weight is complicated and multisided. Researchers have well-documented the impact of these negative attitudes and stereotypes on the experiences of individuals with obesity within education, health care, employment, and within interpersonal relationships, as well as the negative impact on their physical and psychological wellness ([15] [28]). Together, these research results serve as the foundation for positioning weight bias as a social justice issue ([29]). Below we explain how we used our Twitter account not only to inform our research as scientist practitioners, but also to open dialogue through cyber activism through 
giving space for diverse voices and support access to individuals and groups of people who have been excluded or marginalized in dialogue about weight bias.

We have framed the case study and discussion around a selection of contrasting dialogues between the research team and Twitter users, rather than presenting an in-depth analysis of tweets. In this way, we illustrate how social media may be used as a cultural change agent. The selected dialogues illustrate the tensions and divides that are prevalent within the field of weight bias ([13]), whereas others represent stakeholders involved in this sphere, who contributed to the conversation with differing opinions. We hope that, in sharing this case study, we are able to demonstrate that researchers and academics can be involved in social change through dialogue with the immediate social media community, and society at large.

\section{Method: Case Study Research}

Case Study Research (CSR) is a method that seeks to describe and understand the individual (i.e., a culture, organization, group; [30]). For a given inquiry, CSR focuses on a sample of one $(n=1)$ and pursues obtaining data that will help better explain the individual case on a deeper level. In fields such as psychology and education, a significant application of CSR has been in its use of drawing attention to gaps in the literature ([31]). In order to draw attention to an area requiring more research, CSR is helpful in providing audiences with convincing evidence that serious consideration towards the understudied topic is warranted. Thus, our research team began data collection and observation of @UCalgaryBodyBS's Twitter activity, with the intention of studying our experiences of operating this social media account and the feedback received from global Twitter users. Through CSR, we aim to demonstrate our interactions with the Twitter world, and in doing so, will illustrate how we came to understand the process of using social media to disseminate knowledge, and to learn from those we do not traditionally interact with in academic settings.

@UCalgaryBodyBS made frequent use of Twitter communication features such as retweeting and hashtags. These features were used to demonstrate our support for particular content being shared as well as content related to weight bias, the promotion of positive body imagery, and size acceptance.

@UCalgaryBodyBS tweets also featured content-consistent hashtags, such as \#weightbias or \#bodyimage, that could be searched by twitter users, lending itself well to collecting information about a particular topic. Every tweet created by @UCalgaryBodyBS was marked by a hashtag: \#MediaWatch. By including this hashtag in tweets, the team was able to conveniently track the content we shared, which made calculating retweets and likes simpler.

\section{Tweets}

This paper includes the observational analysis of Twitter dialogues, representing different voices, professional backgrounds, and personal biases in relation to 
weight. The excerpts from narratives of passionate individuals communicating publicly online are used to highlight the multiple and often controversial perspectives regarding people with large bodies.

@UCalgaryBodyBS followed 640 accounts, had 497 followers, and produced 1264 tweets. Launched in April 2015, the Twitter account generated global interest, reaching Twitter users in Australia, Brazil, Canada, Germany, Japan, Malaysia, Mexico, Netherlands, Philippines, Spain, Singapore, United Kingdom, and the United States. In order to collect statistics regarding @UCalgaryBodyBS’s impact, the website bitley.com was used. In addition to collecting information on how many times each Tweet was accessed and from what geographic location, this website was used to condense ULR links so that more information could be shared within the 140-character Tweet limit.

\section{Co-Creating an Evolving Social Dialogue: Findings and Discussion}

Through the use of Twitter's tools for efficient communication, @UCalgaryBodyBS was able to elicit feedback from followers, and as such co-created social dialogue. By selectively sharing content via Twitter and engaging with other users, the direction of our Twitter account and research objectives co-evolved based on the interplay between shared events that unfolded via social media. Operating on a many-to-many basis, meaning when social media is being produced by many, rather than being produced by a few commercial or public service agencies, the messages shared are free from censorship ([32]). Rather than being at the control of a gatekeeper, many-to-many communication is shared freely, allowing for social media users to distribute what they want and to respond at will, effectively co-creating social dialogue. As Bruns ([32]) proposed, social media is social only when the medium mediates, and when communication is not fixed by the creator alone; rather, the messages are co-created, and co-evolved based on the interaction between the creator, operators, and users.

\section{Reaching Broader Audiences: Interdisciplinary Dialogue}

Our Twitter account sparked the interest of a range of social groups, which according to Schnitzler et al. ([10]), allows for the visibility of the research profile and team's objective to be raised. @UCalgaryBodyBS has an array of diverse followers including: nurses, medical doctors, dieticians, nutritionists, psychologists, psychiatrists, social workers, occupational therapists, obesity scholars, eating disorder specialists, fat activists, Health at Every Size (HAES) advocates, parents, educators, self proclaimed feminists, eating disorder treatment centers, and fitness enthusiasts. Using Twitter as an informal communication tool allowed us to connect across perspectives and disciplines, share differing positions, and learn from the viewpoints of others. Table 1 demonstrates an open dialogue between @UCalgaryBodyBS, an individual sharing experience living with obesity, HAES and eating disorder activists, and a registered dietitian. 
Table 1. Opening dialogue on Language.

\begin{tabular}{|c|c|c|}
\hline Date & Twitter User & Tweet \\
\hline \multirow[t]{5}{*}{ October 9, 2015} & @UCalgaryBodyBS & $\begin{array}{l}\text { "Obese" "fat" "people living in larger bodies" "people of } \\
\text { size".. Which term do you prefer? \#weight \#language }\end{array}$ \\
\hline & @AdriannaORegan & $\begin{array}{l}\text { @UCalgaryBodyBS How about none of those? Living with } \\
\text { obesity sounds better to my ears personally. }\end{array}$ \\
\hline & @lisajdubreuil & $\begin{array}{l}\text { Any/all that are neutral \& physically descriptive are good } \\
\text { w/me. Nope to "obese" "overweight", they R stigmatizing. }\end{array}$ \\
\hline & @HAEScoach & $\begin{array}{l}\text { @lisajdubreuil @UCalgaryBodyBS I'd like it to say those that } \\
\text { are outside our current narrow cultural norm. Too much to } \\
\text { ask? }\end{array}$ \\
\hline & @MeganCharlish & $\begin{array}{l}\text { @UCalgaryBodyBS I prefer "ppl w/obesity" or } \\
\text { "ppl w/excess body weight" }\end{array}$ \\
\hline
\end{tabular}

Another example below in Table 2 illustrates how individuals from different professional and personal backgrounds engaged in dialogue related to language around weight. The voices included in this example come from @UCalgaryBodyBS, an author, a registered dietitian, a HAES/eating disorder/ addictions specialist, and an un-specified non-professional.

Evidently, people from different fields as well as non-professionals engaging in conversation around weight and language produce diverse perspectives. The range of different views that emerged from this open dialogue demonstrate the tensions in the field regarding language, and also allowed for the visibility of a range of stakeholders interested and involved in weight related issues to be uncovered. Twitter allowed for our research team to network with users worldwide and in real time, and due to the ability to exchange ideas at an exponential speed, the team was constantly reminded of differences, tensions, and conflicts emerging in the field of weight.

\section{Uncovering Tensions through Debate}

In addition to hearing diverse voices, utilizing Twitter allowed for instantaneous feedback from users, either responding directly to @UCalgaryBodyBS's tweets, or retweeting content and adding additional commentary. It was clear that some content shared sparked controversy. The debate sparked by such tweets by @UCalgaryBodyBS uncovered new tensions or highlighted existing tensions that represented hot button issues within the topic of weight bias. Some of the tensions noted online were demonstrated in the form of disagreement, possibly resulting from different professional affiliations and conceptualizations of weight and health, as might be the case illustrated in Table 3 .

The example above demonstrates contrasting views and perspectives towards obesity. In such situations, it was made clear that individuals from professions rooted in the medical model held different perspectives than individuals adopting the HAES approach. Differing perspectives with regard to our understanding of weight bias, including the tensions between researchers from a medical or "weight centric" perspective and those from a "non-weight centric" perspective have previously been discussed in more detail ([13]). Interactions between 
Table 2. Tensions through debate.

\begin{tabular}{|c|c|c|}
\hline Date & Twitter User & Tweet \\
\hline \multirow[t]{6}{*}{$\begin{array}{l}\text { October } 17, \\
2015\end{array}$} & $@ U C a l g a r y B o d y B$ & $\begin{array}{l}\text { Retweeted @MeganCharlish } \\
\text { S The person comes first. TY for reminding everyone of this } \\
\text { @MeganCharlish }\end{array}$ \\
\hline & @MeganCharlish & $\begin{array}{l}\text { @MichaelMossC Hey, Michael...please use person-first } \\
\text { language. Ppl w/obesity and not "obese ppl”. @UCalgaryBodyBS }\end{array}$ \\
\hline & @lisajdubreuil & $\begin{array}{l}\text { @UCalgaryBodyBS @MeganCharlish Sorry, no. Plse do not assume } \\
\text { fat pple want "people first" language. Many find it stigmatizing. 1/2 }\end{array}$ \\
\hline & @lisajdubreuil & $\begin{array}{l}\text { @UCalgaryBodyBS @MeganCharlish Many disabled pple don't like } \\
\text { it either. "People first" language is controversial for many. } 2 / 2\end{array}$ \\
\hline & @lisajdubreuil & $\begin{array}{l}@ \text { DrSuePedersen @UCalgaryBodyBS Telling me I am ill }{ }^{* *} \text { because } \\
\text { of my body size }{ }^{* *} \text { is stigmatizing in and of itself. \#nothelping }\end{array}$ \\
\hline & @maria_angela69 & $\begin{array}{l}\text { @fatnutritionist @UCalgaryBodyBS I don't think fatness is good. } \\
\text { It's a disease :) }\end{array}$ \\
\hline
\end{tabular}

Table 3. Tensions in Language.

\begin{tabular}{|c|c|c|}
\hline Date & Twitter User & Tweet \\
\hline \multirow[t]{3}{*}{$\begin{array}{l}\text { November 5, } \\
2015\end{array}$} & @UCalgaryBodyBS & $\begin{array}{l}\text { \#MediaWatch “Time to act on obesity” video shared at } \\
\text { \#OW2015 bit.ly/20vBciA Check it out! }\end{array}$ \\
\hline & & Retweeted @UCalgaryBodyBS. \\
\hline & @lisajdubreuil & $\begin{array}{l}\text { Nope, I don't need "treatment" because I am fat, \& that } \\
\text { assumption is stigmatizing. \#nothelping \#weightbias \#HAES }\end{array}$ \\
\hline
\end{tabular}

@UCalgaryBodyBS and Twitter users were representative of dialogues between individuals coming from various theoretical orientations and understandings of how to discuss and understand the complexity of body weight. By utilizing Twitter, the team was able to connect individuals who do not regularly interact in practice, whereby tensions were uncovered through dialogue, as can be seen below in Table 4 and Table 5.

The tensions and debate that occurred in discussions with @UCalgaryBodyBS followers most often centered on language, a topic that has been previously identified as a tension between different perspectives in weight bias research ([13]). Some researchers tend to prefer to use the word obesity as well as person-first language (i.e., person with obesity) as a way to demonstrate that having obesity is only one aspect of an individual's overall identity ([33]). Conversely, other researchers have argued that the word obesity serves to pathologize large bodies as diseased, and instead opt to reclaim the word fat as a neutral identity descriptor when discussing weight and when referring to individuals with large bodies (i.e., fat person; [34]). Research investigating the perception of words such as fat and obese as motivating, desirable, blaming, and stigmatizing has demonstrated no clear preference in language among members of the general public ([26]). Such mixed results highlight how no weight term may be free of 
Table 4. Obesity rhetoric.

\begin{tabular}{|c|c|c|}
\hline Date & Twitter User & Tweet \\
\hline October 3, 2015 & @sfkirk & $\begin{array}{l}\text { Key message-focus on health not weight. Sydney } \\
\text { conference aims to change how health-care workers treat } \\
\text { obesity cbc.ca/1.3254776 }\end{array}$ \\
\hline \multirow[t]{2}{*}{ October 6, 2015} & @lisajdubreuil & $\begin{array}{l}\text { @Karen_Patte @UCalgaryBodyBS @sfkirk Focus on } \\
\text { behaviours }{ }^{* *} \text { by not using obesity rhetoric }{ }^{* *} \text {. It's a } \\
\text { stigmatizing framework. } \\
\text { @sfkirk@UCalgaryBodyBS Treat fat people.not “obesity" }\end{array}$ \\
\hline & @lisajdubreuil & $\begin{array}{l}\text { There is no illness only fat people get. Treat the illness, not } \\
\text { the body size. }\end{array}$ \\
\hline
\end{tabular}

Table 5. Diverse perspectives on Language.

\begin{tabular}{|c|c|c|}
\hline Date & Twitter User & Tweet \\
\hline $\begin{array}{l}\text { October 15, } \\
2015\end{array}$ & @UCalgaryBodyBS & $\begin{array}{l}\text { \#MediaWatch The social function of \#fattalk } \\
\text { bit.ly/1Pji2sa }\end{array}$ \\
\hline & @lisajdubreuil & 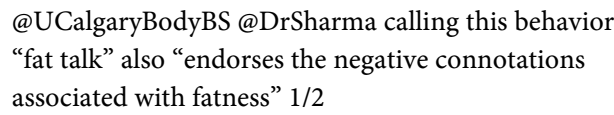 \\
\hline & @lisajdubreuil & 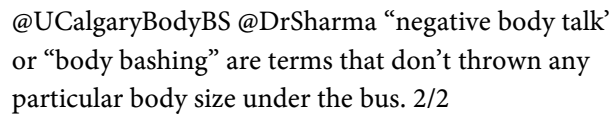 \\
\hline
\end{tabular}

stigma ([26]), which might suggest that greater efforts to increase the awareness of weight stigma are needed ([35]).

\section{Research Impact and Benefits of @UCalgaryBodyBS}

Incorporating Twitter as a source of knowledge mobilization and social networking allowed us to learn and informally exchange information with individuals with diverse perspectives. Such knowledge exchange, collaboration, and innovation is central to interdisciplinary research. Hazel and Onaga ([36]) proposed that social scientists should collaborate with those with lived experience of the phenomenon under study, as working closely with individuals impacted by the topic can provide valuable insight. In line with Hazel and Onaga's ([36]) suggestion, @UCalgaryBodyBS’s weekly Tweets encouraged dialogue among a wide spread of social and professional groups and included individuals with personal experiences with weight bias. Twitter enabled the researchers as well as those engaged in dialogue with @UCalgaryBodyBS to appreciate the varied viewpoints, attitudes, and ideas that resulted from Twitter users' diverse training, professional, and personal experiences. Learning and hearing from others in this casual way informed the team's understanding, and was incorporated into more formal dissemination, showcasing the multiple perspectives and their respective approaches to weight. Ultimately, utilizing twitter to disseminate research was a practical approach when connecting with a wider audience.

When the benefits of research are acknowledged outside of academia, research is said to have broader impact ([10] [37]). Further, Powell ([2]) positioned intellectual property as a cultural production. This production should be shared 
across cultural boundaries rather than being solely kept for the consumption of academics. In the case of @UCalgaryBodyBS, ideas and information produced within an interdisciplinary academic group were translated to informal, easy to consume communication that sparked discussion. Information related to weight bias and other weight related issues (i.e., fat acceptance, positive body image, health at every size) were discussed online with worldwide Twitter users, and in turn, our interdisciplinary team's understanding of this phenomenon was challenged and deepened as a result. Researchers interested in social media's practicality for knowledge dissemination should keep in mind that social media is more than a tool for gathering information, rather it goes beyond this function. Researchers can disseminate their findings on platforms such as twitter in addition to publishing in research journals, and thus participate in dissemination extending across traditional boundaries.

Further, the open dialogue occurring on Twitter and specifically interactions involving @UCalgaryBodyBS may have the potential to contribute to social change or a growing awareness of weight bias as a social justice issue. Debates observed online had the potential to broaden individuals' perspectives and to increase willingness to engage in conversation and collaboration with others from different backgrounds and viewpoints. In addition, by disseminating information that is sometimes only available within academia to the public sphere, the topics tweeted about were made more available to the general public, where discussion of body diversity and size acceptance remains difficult to find.

\section{Limitations and Directions for Future Research}

Although arguments have been made for the widespread use of Twitter, it is important to consider that researchers might not be reaching those who do not have regular access to electronic devices and/or the internet. As such, although Twitter can be used in the cycle of research in many ways, its use beyond increasing knowledge mobilization remains limited ([1]). Further, although @UCalgaryBodyBS was an active Twitter account and tweets were posted at least once per day during Mondays-Fridays, the account did not receive as many followers as hoped for. Though having approximately 500 followers was helpful and did often spark conversations and debates, greater impact on the community may have been observed if there were more followers and greater dialogue among members of the general public.

Based on these limitations, there are several directions researchers could take in the future. One consideration may be for researchers to examine hot-button topics, purposefully creating dialogue from different perspectives and examining whether it is helpful or unhelpful to be contributing to the topic with such charged, salient tweets. Perhaps by intentionally sharing and stirring up conversation about weight among different groups it would further promote weight bias as a topic that requires more attention, not only in the academic world but also within the public sphere. Further, researchers could investigate Twitter as a 
tool for reaching perspectives beyond professionals or for reaching across professional boundaries. An examination into this could provide insight for bridging divergent perspectives related to weight bias, uniting individuals under the common goal of increasing size acceptance and recognizing weight bias as a social justice issue.

\section{Conclusion}

Our interdisciplinary team's desire to reach further than academic perspectives on weight bias was the initial reason social media was considered. Soon into our Twitter endeavor however, we saw how Twitter had the capacity to open social dialogue, spark debate and controversy, and connect us and others to diverse individuals and groups who may not have connected if it was not online. Thus, Twitter served as a medium between us as researchers, attempting to demonstrate weight bias as a social justice issue, to community members, professional bodies, and lay people, all of whom had unique opinions on the topic. The use of social media and Twitter specifically, became more than a tool to inform the research life cycle and disseminate to a global audience at real time speed, through supporting our research team to become social activists, in our case for addressing weight bias.

\section{Acknowledgements}

We would like to thank Dr. Monica Sesma for her contributions to the conceptualization of this article.

\section{References}

[1] Holmberg, K. and Thelwall, M. (2014) Disciplinary Differences in Twitter Scholarly Communication. Scientometrics, 101, 1027-1042. https://doi.org/10.1007/s11192-014-1229-3

[2] Powell, A.B. (2015) Open Culture and Innovation: Integrating Knowledge across Boundaries. Media, Culture \& Society, 37, 376-393. https://doi.org/10.1177/0163443714567169

[3] Tri-Agency Open Access Policy on Publications (2016). http://www.science.gc.ca/eic/site/063.nsf/eng/h_F6765465.html?OpenDocument

[4] Rowlands, I., Nicholas, D., Russell, B., Canty, N. and Watkinson, A. (2011) Social Media Use in the Research Workflow. Learned Publishing, 24, 183-195. https://doi.org/10.1087/20110306

[5] Nichols, N., Phipps, D.J., Provençal, J. and Hewitt, A. (2013) Knowledge Mobilization, Collaboration, and Social Innovation: Leveraging Investments in Higher Education. Canadian Journal of Nonprofit and Social Economy Research, 4, 25-42. https://doi.org/10.22230/cjnser.2013v4n1a126

[6] Biswas, A. and Kirchherr, J. (2015) Citations Are Not Enough: Academic Promotion Panels Must Take into Account a Scholar's Presence in Popular Media. http://blogs.lse.ac.uk/impactofsocialsciences/2015/04/09/academic-promotion-schol ars-popular-media/

[7] Burke-Garcia, A. and Scally, G. (2014) Trending Now: Future Directions in Digital 
Media for the Public Health Sector. Journal of Public Health, 36, 527-534. https://doi.org/10.1093/pubmed/fdt125

[8] Jensen, K.B. (2015) What's Social about Social me dia? Social Media + Society, 1, 205630511557887. https://doi.org/10.1332/174426413X671086

[9] Murdock, A., Shariff, R. and Wilding, K. (2013) Knowledge Exchange between Academia and the Third Sector. Evidence \& Policy: A Journal of Research, Debate and Practice, 9, 419-430.

[10] Schnitzler, K., Davies, N., Ross, F. and Harris, R. (2016) Using Twitter ${ }^{\mathrm{Tm}}$ to Drive Research Impact: A Discussion of Strategies, Opportunities and Challenges. International Journal of Nursing Studies, 59, 15-26. https://doi.org/10.1016/j.ijnurstu.2016.02.004

[11] Power, A. (2015) Twitter's Potential to Enhance Professional Networking. British Journal of Midwifery, 23, 65-67. https://doi.org/10.12968/bjom.2015.23.1.65

[12] Boyd, D.M. and Ellison, N.B. (2007) Social Network Sites: Definition, History, and Scholarship. Journal of Computer-Mediated Communication, 13, 210-230. https://doi.org/10.1111/j.1083-6101.2007.00393.x

[13] Nutter, S., Kassan, A., Russell-Mayhew, S., Sesma-Vazquez, M., Lund, D.E., Arthur, N. and Williams, E. (2016) Positioning of Weight Bias: Moving towards Social Justice. Journal of Obesity, 2016, Article ID: 3753650. https://doi.org/10.1155/2016/3753650

[14] Chou, W.S., Prestin, A. and Kunath, S. (2014) Obesity in Social Media: A Mixed Methods Analysis. Translational Behavioral Medicine, 4, 314-323. https://doi.org/10.1007/s13142-014-0256-1

[15] Carels, R.A. and Latner, J. (2016) Weight Stigma and Eating Behaviors: An Introduction to the Special Issue. Appetite, 102, 1-2. https://doi.org/10.1016/j.appet.2016.03.001

[16] Crandall, C.S. (1994) Prejudice against Fat People: Ideology and Self-Interest. Journal of Personality and Social Psychology, 66, 882-894. https://doi.org/10.1037/0022-3514.66.5.882

[17] Mussap, A.J., Manger, E. and Gold, R.S. (2016) Weight-Control Effort Can Increase Obesity Stereotyping. Personality and Individual Differences, 88, 178-181. https://doi.org/10.1016/j.paid.2015.09.014

[18] Stewart, J. (2014) The School Counsellor's Role in Promoting Social Justice for Refugee and Immigrant Children. Canadian Journal of Counselling and Psychotherapyl Revue Canadienne de Counseling et de Psychothérapie, 48, 251-269. http://cjc-rcc.ucalgary.ca/cjc/index.php/rcc/article/view/2722

[19] Andreyeva, T., Puhl, R.M. and Brownell, K.D. (2008) Changes in Perceived Weight Discrimination among Americans, 1995-1996 through 2004-2006. Obesity, 16, 1129-1134. https://doi.org/10.1038/oby.2008.35

[20] Phelan, S.M., Burgess, D.J., Yeazel, M.W., Hellerstedt, W.L., Griffin, J.M. and Ryn, M. (2015) Impact of Weight Bias and Stigma on Quality of Care and Outcomes for Patients with Obesity. Obesity Reviews, 16, 319-326.

https://doi.org/10.1111/obr.12266

[21] Kenney, E.L., Gortmaker, S.L., Davison, K.K. and Bryn Austin, S. (2015) The Academic Penalty for Gaining Weight: A Longitudinal, Change-in-Change Analysis of BMI and Perceived Academic Ability in Middle School Students. International Journal of Obesity, 39, 1408-1413. https://doi.org/10.1038/ijo.2015.88

[22] Vanhove, A. and Gordon, R.A. (2014) Weight Discimination in the Workplace: A 
Meta-Analytic Examination of the Relationship Weight and Work-Related Outcomes. Journal of Applied Social Psychology, 44, 12-22. https://doi.org/10.1111/jasp.12193

[23] Puhl, R.M., Luedicke, J. and Heuer, C. (2011) Weight-Based Victimization towards Overweight Adolescents: Observations and Reactions of Peers. Journal of School Health, 81, 696-703. https://doi.org/10.1111/j.1746-1561.2011.00646.x

[24] Ata, R.N. and Thompson, J.K. (2010) Weight Bias in the Media: A Review of Recent Research. Obesity Facts, 3, 41-46. https://doi.org/10.1159/000276547

[25] Lydecker, J.A., Cotter, E.W., Palmberg, A.A., Simpson, C., Kwitowski, M., White, K. and Mazzeo, S.E. (2016) Does This Tweet Make Me Look Fat? A Content Analysis of Weight Stigma on Twitter. Eating and Weight Disorders-Studies on Anorexia, Bulimia and Obesity, 21, 229-235. https://doi.org/10.1007/s40519-016-0272-x

[26] Puhl, R.M., Petersen, J.L., DePierre, J.A. and Luedicke, J. (2013) Headless, Hungry, and Unhealthy: A Video Content Analysis of Obese Persons Portrayed in Online News. Journal of Health Communication, 18, 686-702.

https://doi.org/10.1080/10810730.2012.743631

[27] Vartanian, L.R. and Smyth, J.M. (2013) Primum Non Nocere: Obesity Stigma and Public Health. Bioethical Inquiry, 10, 49-57. https://doi.org/10.1007/s11673-012-9412-9

[28] Puhl, R.M. and Heuer, C.A. (2009) The Stigma of Obesity: A Review and Update. Obesity, 17, 941-964. https://doi.org/10.1038/oby.2008.636

[29] Nutter, S., Russell-Mayhew, S., Arthur, N., and Ellard, J.H. (2018) Weight Bias as a Social Justice Issue: A Call for Dialogue. Canadian Psychology/Psychologie Canadienne, 59, 89-99. https://doi.org/10.1037/cap0000125

[30] Woodside, A.G. and Ebrary, I. (2010) Case Study Research: Theory, Methods, Practice. Emerald Group Publication, Bingley.

[31] McLeod, J. and British Association for Counselling and Psychotherapy (2010) Case Study Research in Counselling and Psychotherapy. SAGE, London, Los Angeles.

[32] Bruns, A. (2015) Making Sense of Society through Social Media. Social Media + Society, 1, 1-2.

[33] Forhan, M. and Salas, X.R. (2013) Inequities in Healthcare: A Review of Bias and Discrimination in Obesity Treatment. Canadian Journal of Diabetes, 37, 205-209. https://doi.org/10.1016/j.jcjd.2013.03.362

[34] Cooper, C. (2010) Fat Studies: Mapping the Field. Sociology Compass, 4, 1020-1034. https://doi.org/10.1111/j.1751-9020.2010.00336.x

[35] Warin, M.J. and Gunson, J.S. (2013) The Weight of the Word: Knowing Silences in Obesity Research. Qualitative Health Research, 23, 1686-1696. https://doi.org/10.1177/1049732313509894

[36] Hazel, K.L. and Onaga, E. (2003) Experimental Social Innovation and Dissemination: The Promise and Its Delivery. American Journal of Community Psychology, 32, 285-294. https://doi.org/10.1023/B:AJCP.0000004748.50885.2e

[37] McKenna, H., Daly, J., Davidson, P., Duffield, C. and Jackson, D. (2012) RAE and ERA-Spot the Difference. International Journal of Nursing Studies, 49, 375-377. https://doi.org/10.1016/j.ijnurstu.2011.11.013 